\title{
On generalized difference lacunary statistical convergence in a paranormed space
}

Selma Altundağ*

\section{"Correspondence:}

scaylan@sakarya.edu.tr

Mathematics Department, Faculty

of Arts and Science, Sakarya

University, Sakarya, 54187, Turkey

\begin{abstract}
In this article, we introduce the concept of $\Delta^{m}$-lacunary statistical convergence and $\Delta^{m}$-lacunary strong convergence in a paranormed space. Also, we establish some connections between these concepts.
\end{abstract}

\section{Introduction}

In order to extend convergence of sequences, the notion of statistical convergence was introduced by Fast [1] and Steinhaus [2] and several generalizations and applications of this concept have been investigated by various authors [3, 4]. This notion was studied in normed spaces by Kolk [5], in locally convex Hausdorff topological spaces by Maddox [6], in topological Hausdorff groups by Çakallı [7] and in probabilistic normed space by Karakuş [8]. Recently, Alotaibi and Alroqi [9] extended this notion in paranormed spaces.

In this article, we study the concept of statistical convergence from difference sequence spaces which are defined over paranormed space.

\section{Preliminaries and definitions}

Let $K$ be a subset of the set of natural numbers $\mathbb{N}$. Then the asymptotic density of $K$ denoted by $\delta(K)=\lim _{n} \frac{1}{n}|\{k \leq n: k \in K\}|$, where the vertical bars denote the cardinality of the enclosed set in [10].

A number sequence $x=\left(x_{k}\right)$ is said to be statistically convergent to the number $L$ if for each $\varepsilon>0$, the set $K(\varepsilon)=\left\{k \leq n:\left|x_{k}-L\right| \geq \varepsilon\right\}$ has asymptotic density zero, i.e.,

$$
\lim _{n} \frac{1}{n}\left|\left\{k \leq n:\left|x_{k}-L\right| \geq \varepsilon\right\}\right|=0
$$

In this case we write $s t$ - $\lim x=L$. This concept was studied by $[11,12]$.

By a lacunary $\theta=\left(k_{r}\right) ; r=0,1,2, \ldots$, where $k_{0}=0$, we shall mean an increasing sequence of non-negative integers with $k_{r}-k_{r-1} \rightarrow \infty$ as $r \rightarrow \infty$. The intervals determined by $\theta$ will be denoted by $I_{r}=\left(k_{r-1}, k_{r}\right]$ and $h_{r}=k_{r}-k_{r-1}$. The ratio $\frac{k_{r}}{k_{r-1}}$ will be denoted by $q_{r}$.

The notion of difference sequence space $X(\Delta)$ was introduced by Kizmaz [13] as follows:

$$
X(\Delta)=\left\{x=\left(x_{k}\right):\left(\Delta x_{k}\right) \in X\right\}
$$

for $X=l_{\infty}, c, c_{0}$, where $\Delta x_{k}=x_{k}-x_{k+1}$ for all $k \in \mathbb{N}$.

\section{Springer}

(0) 2013 Altundağ; licensee Springer. This is an Open Access article distributed under the terms of the Creative Commons Attribution License (http://creativecommons.org/licenses/by/2.0), which permits unrestricted use, distribution, and reproduction in any medium, provided the original work is properly cited. 
The notion of difference sequence spaces was further generalized by Et and Çolak [14] as follows:

$$
X\left(\Delta^{m}\right)=\left\{x=\left(x_{k}\right) \in w:\left(\Delta^{m} x_{k}\right) \in X\right\}
$$

for $X=l_{\infty}, c$ and $c_{0}$, where $m \in \mathbb{N}, \Delta^{m} x_{k}=\Delta^{m-1} x_{k}-\Delta^{m-1} x_{k+1}, \Delta^{0} x_{k}=x_{k}$.

The sequence $x$ is said to be $\Delta^{m}$-statistically convergent to the number $L$ provided that for each $\varepsilon>0$,

$$
\lim _{n} \frac{1}{n}\left|\left\{k \leq n:\left|\Delta^{m} x_{k}-L\right| \geq \varepsilon\right\}\right|=0 .
$$

The set of all $\Delta^{m}$-statistically convergent sequences was denoted by $S\left(\Delta^{m}\right)$ in [15].

Furthermore, this notion was studied in $[16,17]$.

A paranorm is a function $g: X \rightarrow \mathbb{R}$ defined on a linear space $X$ such that for all $x, y, z \in X$,

(i) $g(x)=0$ if $x=\theta$;

(ii) $g(-x)=g(x)$;

(iii) $g(x+y) \leq g(x)+g(y)$;

(iv) If $\left(\alpha_{n}\right)$ is a sequence of scalars with $\alpha_{n} \longrightarrow \alpha_{0}(n \longrightarrow \infty)$ and $x_{n}, a \in X$ with $x_{n} \longrightarrow a$ $(n \longrightarrow \infty)$ in the sense that $g\left(x_{n}-a\right) \longrightarrow 0(n \longrightarrow \infty)$, then $\alpha_{n} x_{n} \longrightarrow \alpha_{0} a$

$(n \longrightarrow \infty)$, in the sense that $g\left(\alpha_{n} x_{n}-\alpha_{0} a\right) \longrightarrow 0(n \longrightarrow \infty)$.

A paranorm $g$ for which $g(x)=0$ implies $x=\theta$ is called a total paranorm on $X$ and the pair $(X, g)$ is called a total paranormed space.

Note that each seminorm (norm) $p$ on $X$ is a paranorm (total) but converse need not be true.

The concept of paranorm is a generalization of absolute value [18].

A modulus function $f$ is a function from $[0, \infty)$ to $[0, \infty)$ such that

(i) $f(x)=0$ if and only if $x=0$;

(ii) $f(x+y) \leq f(x)+f(y)$ for all $x, y \geq 0$;

(iii) $f$ increasing;

(iv) $f$ is continuous from at the right zero.

Since $|f(x)-f(y)| \leq f(|x-y|)$, it follows from condition (iv) that $f$ is continuous on $[0, \infty)$. Furthermore, we have $f(n x) \leq n f(x)$ for all $n \in \mathbb{N}$ from condition (ii) and so

$$
f(x)=f\left(n x \frac{1}{n}\right) \leq n f\left(\frac{x}{n}\right) .
$$

Hence, for all $n \in \mathbb{N}$,

$$
\frac{1}{n} f(x) \leq f\left(\frac{x}{n}\right)
$$

A modulus may be bounded or unbounded. For example, $f(x)=x^{p}$ for $0<p \leq 1$ is unbounded, but $f(x)=\frac{x}{1+x}$ is bounded. Ruckle [19] used the idea of a modulus function $f$ to construct a class of FK spaces

$$
L(f)=\left\{x=\left(x_{k}\right): \sum f\left(\left|x_{k}\right|\right)<\infty\right\} .
$$


In [9], the notion of statistical convergence was defined in a paranormed space.

Definition 2.1 A sequence $x=\left(x_{k}\right)$ is said to be statistically convergent to the number $L$ in $(X, g)$ if for each $\varepsilon>0$,

$$
\lim _{n} \frac{1}{n}\left|\left\{k \leq n: g\left(x_{k}-L\right) \geq \varepsilon\right\}\right|=0 .
$$

In this case, we write $g(s t)-\lim x=L$. We denote the set of all $g(s t)$-convergent sequences by $S_{g}[9]$.

Definition 2.2 A sequence $x=\left(x_{k}\right)$ is said to be strongly $p$-Cesaro summable $(0<p<\infty)$ to the limit $L$ in $(X, g)$ if

$$
\lim _{n} \frac{1}{n} \sum_{j=1}^{n}\left(g\left(x_{j}-L\right)\right)^{p}=0,
$$

and we write it as $x_{k} \longrightarrow L\left(\left[C_{1}, g\right]_{p}\right)$. In this case, $L$ is called the $\left[C_{1}, g\right]_{p}$-lim it of $x$ [9].

In this article, we shall study the concept of $\Delta^{m}$-lacunary statistical convergence, $\Delta^{m}$-lacunary strong convergence and $\Delta^{m}$-lacunary strong convergence with respect to a modulus function in a paranormed space.

\section{Generalized difference statistical convergence in a paranormed space}

Definition 3.1 A sequence $x=\left(x_{k}\right)$ is said to be $\Delta^{m}$-statistically convergent to the number $L$ in $(X, g)$ if for each $\varepsilon>0$,

$$
\lim _{n} \frac{1}{n}\left|\left\{k \leq n: g\left(\Delta^{m} x_{k}-L\right) \geq \varepsilon\right\}\right|=0 .
$$

In this case, we write $S_{g}\left(\Delta^{m}\right)$-lim $x=L$. We denote the set of all $\Delta^{m}$-statistically convergent sequences in $(X, g)$ by $S_{g}\left(\Delta^{m}\right)$.

Definition 3.2 Let $\theta$ be a lacunary sequence. A sequence $x=\left(x_{k}\right)$ is said to be $\Delta^{m}$-lacunary statistically convergent to the number $L$ in $(X, g)$ if for each $\varepsilon>0$,

$$
\lim _{r} \frac{1}{h_{r}}\left|\left\{k \in I_{r}: g\left(\Delta^{m} x_{k}-L\right) \geq \varepsilon\right\}\right|=0 .
$$

In this case, we write $S_{g}^{\theta}\left(\Delta^{m}\right)$-lim $x=L$. We denote the set of all $\Delta^{m}$-lacunary statistically convergent sequences in $(X, g)$ by $S_{g}^{\theta}\left(\Delta^{m}\right)$.

Definition 3.3 A sequence $x=\left(x_{k}\right)$ is said to be strongly $\Delta^{m}$-Cesaro summable to the limit $L$ in $(X, g)$ if

$$
\lim _{n} \frac{1}{n} \sum_{k=1}^{n}\left(g\left(\Delta^{m} x_{k}-L\right)\right)=0,
$$

and we write it as $x_{k} \longrightarrow L\left(\left|\sigma_{1}\right|_{g}\left(\Delta^{m}\right)\right)$. In this case $L$ is called the $\left|\sigma_{1}\right|_{g}\left(\Delta^{m}\right)$-lim of $x$. 
Definition 3.4 A sequence $x=\left(x_{k}\right)$ is said to be strongly $\Delta^{m}$-lacunary strongly summable to the limit $L$ in $(X, g)$ if

$$
\lim _{r} \frac{1}{h_{r}} \sum_{k \in I_{r}}\left(g\left(\Delta^{m} x_{k}-L\right)\right)=0
$$

and we write it as $x_{k} \longrightarrow L\left(N_{g}^{\theta}\left(\Delta^{m}\right)\right)$. In this case $L$ is called the $N_{g}^{\theta}\left(\Delta^{m}\right)$-lim of $x$.

Theorem 3.1 Let $\theta$ be a lacunary sequence and $(X, g)$ be a paranormed space. Then

(i) If $x_{k} \longrightarrow L\left(N_{g}^{\theta}\left(\Delta^{m}\right)\right)$, then $x_{k} \longrightarrow L\left(S_{g}^{\theta}\left(\Delta^{m}\right)\right)$ and the inclusion is strict;

(ii) If $x$ is a $\Delta^{m}$-bounded sequence and $x_{k} \longrightarrow L\left(S_{g}^{\theta}\left(\Delta^{m}\right)\right)$, then $x_{k} \longrightarrow L\left(N_{g}^{\theta}\left(\Delta^{m}\right)\right)$;

(iii) $l_{g}^{\infty}\left(\Delta^{m}\right) \cap S_{g}^{\theta}\left(\Delta^{m}\right)=l_{g}^{\infty}\left(\Delta^{m}\right) \cap N_{g}^{\theta}\left(\Delta^{m}\right)$.

Proof (i) If $\varepsilon>0$ and $x_{k} \rightarrow L\left(N_{g}^{\theta}\left(\Delta^{m}\right)\right)$, we can write

$$
\sum_{k \in I_{r}} g\left(\Delta^{m} x_{k}-L\right) \geq \sum_{\substack{k \in I_{r} \\ g\left(\Delta^{m} x_{k}-L\right) \geq \varepsilon}} g\left(\Delta^{m} x_{k}-L\right) \geq \varepsilon\left|\left\{k \in I_{r}: g\left(\Delta^{m} x_{k}-L\right) \geq \varepsilon\right\}\right|,
$$

which yields the result.

In order to prove that the inclusion $N_{g}^{\theta}\left(\Delta^{m}\right) \subset S_{g}^{\theta}\left(\Delta^{m}\right)$ is proper, let $\theta$ be given and $X=N_{0}^{\theta}\left(\Delta, \frac{1}{h_{r}}\right)=\left\{x=\left(x_{k}\right):\left|\frac{1}{h_{r}} \sum_{k \in I_{r}} \Delta x_{k}\right|^{\frac{1}{h_{r}}} \rightarrow 0, r \rightarrow \infty\right\}$ with the paranorm $g(x)=\left|x_{1}\right|+$ $\sup _{r}\left|\frac{1}{h_{r}} \sum_{k \in I_{r}} \Delta x_{k}\right|^{\frac{1}{h_{r}}}$. Define $x=\left(x_{k}\right)$ to be $2 h_{r} 1^{h_{r}}$ at the first term in $I_{r}$ for every $r \geq 1$, $x_{k}=h_{r}\left(1^{h_{r}}-2^{h_{r}}-\cdots-(k-1)^{h_{r}}\right)$ between the second term and $\left(\left[\sqrt{h_{r}}\right]+1\right)$ th term in $I_{r}$, $x_{k}=h_{r}\left(1^{h_{r}}-2^{h_{r}}-\cdots-\left(\left[\sqrt{h_{r}}\right]\right)^{h_{r}}\right)$ at the $\left(\left[\sqrt{h_{r}}\right]+2\right)$ th term in $I_{r}$ and $x_{k}=0$ otherwise.

We see that

$$
\Delta x_{k}= \begin{cases}h_{r} 1^{h_{r}}, h_{r} 2^{h_{r}}, \ldots, h_{r}\left[\sqrt{h_{r}}\right]^{h_{r}}, & \text { at the first }\left[\sqrt{h_{r}}\right] \text { integers in } I_{r}, \\ 0, & \text { otherwise. }\end{cases}
$$

and

$$
g\left(\Delta x_{k}\right)= \begin{cases}1,2, \ldots,\left[\sqrt{h_{r}}\right], & \text { at the first }\left[\sqrt{h_{r}}\right] \text { integers in } I_{r}, \\ 0, & \text { otherwise. }\end{cases}
$$

Note that $x$ is not $\Delta$-bounded in $(X, g)$. We have, for every $\varepsilon>0$,

$$
\left.\frac{1}{h_{r}} \mid\left\{k \in I_{r}: g\left(\Delta x_{k}\right) \geq \varepsilon\right)\right\} \mid=\frac{\left[\sqrt{h_{r}}\right]}{h_{r}} \rightarrow 0
$$

as $r \rightarrow \infty$, i.e., $x_{k} \rightarrow 0\left(S_{g}^{\theta}(\Delta)\right)$. On the other hand,

$$
\frac{1}{h_{r}} \sum_{k \in I_{r}} g\left(\Delta x_{k}\right)=\frac{1}{h_{r}} \frac{\left[\sqrt{h_{r}}\right]\left(\left[\sqrt{h_{r}}\right]+1\right)}{2} \rightarrow \frac{1}{2} \neq 0
$$

hence $x_{k} \nrightarrow 0\left(N_{g}^{\theta}(\Delta)\right)$. 
(ii) Suppose that $x_{k} \rightarrow L\left(S_{g}^{\theta}\left(\Delta^{m}\right)\right)$ and say $g\left(\Delta^{m} x_{k}-L\right) \leq M$ for all $k$. Given $\varepsilon>0$, we get

$$
\begin{aligned}
\frac{1}{h_{r}} \sum_{k \in I_{r}} g\left(\Delta^{m} x_{k}-L\right) & =\frac{1}{h_{r}} \sum_{\substack{k \in I_{r} \\
g\left(\Delta^{m} x_{k}-L\right) \geq \varepsilon}} g\left(\Delta^{m} x_{k}-L\right)+\frac{1}{h_{r}} \sum_{\substack{k \in I_{r} \\
g\left(\Delta^{m} x_{k}-L\right)<\varepsilon}} g\left(\Delta^{m} x_{k}-L\right) \\
& \leq \frac{M}{h_{r}}\left|\left\{k \in I_{r}: g\left(\Delta^{m} x_{k}-L\right) \geq \varepsilon\right\}\right|+\varepsilon,
\end{aligned}
$$

from which the result follows.

(iii) This is an immediate consequence of (i) and (ii).

Corollary 3.1 If $x_{k} \rightarrow L\left(\left|\sigma_{1}\right|_{g}\left(\Delta^{m}\right)\right)$, then $x_{k} \rightarrow L\left(S_{g}\left(\Delta^{m}\right)\right)$. If $x \in l_{g}^{\infty}\left(\Delta^{m}\right)$ and if $x_{k} \rightarrow$ $L\left(S_{g}\left(\Delta^{m}\right)\right)$, then $x_{k} \rightarrow L\left(\left|\sigma_{1}\right|_{g}\left(\Delta^{m}\right)\right)$.

Theorem 3.2 Let $\theta$ be a lacunary sequence and $(X, g)$ be a paranormed space, then $S_{g}^{\theta}\left(\Delta^{m}\right)=S_{g}\left(\Delta^{m}\right)$ if and only if $1<\liminf _{r} q_{r} \leq \lim \sup _{r} q_{r}<\infty$.

Proof Suppose that liminf $q_{r}>1$, then there exists a $\delta>0$ such that $q_{r} \geq 1+\delta$ for sufficiently large $r$, which implies that

$$
\frac{h_{r}}{k_{r}} \geq \frac{\delta}{1+\delta}
$$

If $x_{k} \rightarrow L\left(S_{g}\left(\Delta^{m}\right)\right)$, then for every $\varepsilon>0$ and sufficiently large $r$, we have

$$
\begin{aligned}
\frac{1}{k_{r}}\left|\left\{k \leq k_{r}: g\left(\Delta^{m} x_{k}-L\right) \geq \varepsilon\right\}\right| & \geq \frac{1}{k_{r}}\left|\left\{k \in I_{r}: g\left(\Delta^{m} x_{k}-L\right) \geq \varepsilon\right\}\right| \\
& \geq \frac{\delta}{1+\delta} \frac{1}{h_{r}}\left|\left\{k \in I_{r}: g\left(\Delta^{m} x_{k}-L\right) \geq \varepsilon\right\}\right|,
\end{aligned}
$$

which proves the $S_{g}\left(\Delta^{m}\right) \subset S_{g}^{\theta}\left(\Delta^{m}\right)$.

Conversely, suppose that $\liminf q_{r}=1$. Since $\theta$ is lacunary, we can select a subsequence $\left(k_{r_{j}}\right)$ of $\theta$ satisfying $\frac{k_{r_{j}}}{k_{r_{j}-1}}<1+\frac{1}{j}$ and $\frac{k_{r_{j}-1}}{k_{r_{(j-1)}}}>j$, where $r_{j} \geq r_{j-1}+2$ and $X=$ $N_{0}^{\theta}\left(\Delta, \frac{1}{h_{r}}\right)=\left\{x=\left(x_{k}\right):\left|\frac{1}{h_{r}} \sum_{k \in I_{r}} \Delta x_{k}\right|^{\frac{1}{h_{r}}} \rightarrow 0, r \rightarrow \infty\right\}$ with the paranorm $g(x)=\left|x_{1}\right|+$ $\sup _{r}\left|\frac{1}{h_{r}} \sum_{k \in I_{r}} \Delta x_{k}\right|^{\frac{1}{h_{r}}}$.

Now define a sequence by

$$
\Delta x_{k}= \begin{cases}h_{r}+k, & k \in I_{r_{(j)}}, j=1,2, \ldots \\ 0, & \text { otherwise. }\end{cases}
$$

We can see that

$$
g\left(\Delta x_{k}\right)= \begin{cases}1, & k \in I_{r_{(j)}}, j=1,2, \ldots \\ 0, & \text { otherwise }\end{cases}
$$

and hence $x$ is $\Delta$-bounded in $(X, g)$. 
We can see that $x \notin N_{g}^{\theta}\left(\Delta^{m}\right)$, but $x \in \sigma_{g}^{1}\left(\Delta^{m}\right)$. Theorem 3.1(ii) implies that $x \notin S_{g}^{\theta}\left(\Delta^{m}\right)$, but it follows from Corollary 3.1 that $x \in S_{g}\left(\Delta^{m}\right)$. Hence $S_{g}\left(\Delta^{m}\right) \not \subset S_{g}^{\theta}\left(\Delta^{m}\right)$ and $S_{g}\left(\Delta^{m}\right) \subset$ $S_{g}^{\theta}\left(\Delta^{m}\right)$ implies that $\liminf q_{r}>1$.

To show for any lacunary sequence $\theta, S_{g}^{\theta}\left(\Delta^{m}\right) \subset S_{g}\left(\Delta^{m}\right)$ implies $\lim \sup q_{r}<\infty$, the same technique of Lemma 3 of [20] can be used. Now suppose that $\lim \sup q_{r}=\infty$. Consider the same space defined above and the sequence defined by

$$
\Delta x_{i}= \begin{cases}h_{r}+i, & k_{r_{j}-1}<i \leq 2 k_{r_{j}-1}, j=1,2, \ldots \\ 0, & \text { otherwise. }\end{cases}
$$

Then we get

$$
g\left(\Delta x_{i}\right)= \begin{cases}1, & k_{r_{j}-1}<i \leq 2 k_{r_{j}-1}, j=1,2, \ldots \\ 0, & \text { otherwise }\end{cases}
$$

Then $x \in N_{g}^{\theta}\left(\Delta^{m}\right)$, but $x \notin \sigma_{g}^{1}\left(\Delta^{m}\right)$. By Theorem 3.1(i) we conclude that $x \in S_{g}^{\theta}\left(\Delta^{m}\right)$, but by Corollary 3.1 that $x \notin S_{g}\left(\Delta^{m}\right)$. Hence, $S_{g}^{\theta}\left(\Delta^{m}\right) \not \subset S_{g}\left(\Delta^{m}\right)$. This completes the proof.

Definition 3.5 Let $f$ be a modulus function. Then a sequence $x=\left(x_{k}\right)$ is lacunary strongly $p$-Cesaro summable to $L$ with respect to $f$ in $(X, g)$ if

$$
\lim _{r} \frac{1}{h_{r}} \sum_{k \in I_{r}}\left[f\left(g\left(\Delta^{m} x_{k}-L\right)\right)\right]^{p_{k}}=0 .
$$

In this case, we write $x_{k} \rightarrow L\left(N_{g}^{\theta}\left(f, \Delta^{m}, p\right)\right)$. If we take $p_{k}=1$ for all $k \in \mathbb{N}$, we say $x_{k} \rightarrow$ $L\left(N_{g}^{\theta}\left(f, \Delta^{m}\right)\right)$.

Lemma 3.1 Let $f$ be a modulus function and let $0<\delta<1$. Then for each $x>\delta$ we have $f(x) \leq 2 f(1) \delta^{-1} x[21]$.

Theorem 3.3 Let $f$ be a modulus function and $(X, g)$ be a paranormed space. Then $N_{g}^{\theta}\left(\Delta^{m}\right) \subset N_{g}^{\theta}\left(f, \Delta^{m}\right)$.

Proof Let $x \in N_{g}^{\theta}\left(\Delta^{m}\right)$. Then we have $\tau_{r}=\frac{1}{h_{r}} \sum_{k \in I_{r}} g\left(\Delta^{m} x_{k}-L\right) \rightarrow 0$ as $r \rightarrow \infty$ for some $L$. Let $\varepsilon>0$ and choose $\delta$ with $0<\delta<1$ such that $f(u)<\varepsilon$ for $u$ with $0 \leq u \leq \delta$. Then we can write

$$
\begin{aligned}
\frac{1}{h_{r}} \sum_{k \in I_{r}} f\left(g\left(\Delta^{m} x_{k}-L\right)\right)= & \frac{1}{h_{r}} \sum_{\substack{k \in I_{r} \\
g\left(\Delta^{m} x_{k}-L\right) \leq \delta}} f\left(g\left(\Delta^{m} x_{k}-L\right)\right) \\
& +\frac{1}{h_{r}} \sum_{\substack{k \in I_{r} \\
g\left(\Delta^{m} x_{k}-L\right)>\delta}} f\left(g\left(\Delta^{m} x_{k}-L\right)\right) \\
\leq & \frac{1}{h_{r}}\left(h_{r} \delta\right)+\frac{1}{h_{r}} 2 f(1) \delta^{-1} h_{r} \tau_{r}
\end{aligned}
$$

from Lemma 3.1. Therefore $x \in N_{g}^{\theta}\left(f, \Delta^{m}\right)$. 
Theorem 3.4 Let $0<\inf _{k} p_{k} \leq p_{k} \leq \sup _{k} p_{k}<\infty$. Then $S_{g}^{\theta}\left(\Delta^{m}\right)=N_{g}^{\theta}\left(f, \Delta^{m}, p\right)$ if and only iff is bounded.

Proof Following the technique applied for establishing Theorem 3.16 of [22], we can prove the theorem.

\section{Competing interests}

The author declares that they have no competing interests.

\section{Acknowledgements}

Dedicated to Professor Hari M Srivastava.

The author would like to thank the referees for their careful reading of the manuscript and for their helpful suggestions.

Received: 14 December 2012 Accepted: 6 May 2013 Published: 20 May 2013

\section{References}

1. Fast, H: Sur la convergence statistique. Colloq. Math. 2, 241-244 (1951)

2. Steinhaus, H: Sur la convergence ordinaire et la convergence asymptotique. Colloq. Math. 2, 73-74 (1951)

3. Fridy, JA: Lacunary statistical summability. J. Math. Anal. Appl. 173, 497-504 (1993)

4. Mursaleen, M, Mohiuddine, SA: On lacunary statistical convergence with respect to the intuitionistic fuzzy normed space. J. Comput. Appl. Math. 233(2), 142-149 (2009)

5. Kolk, E: The statistical convergence in Banach spaces. Tartu ülik. Toim. 928, 41-52 (1991)

6. Maddox, IJ: Statistical convergence in a locally convex space. Math. Proc. Camb. Philos. Soc. 104, 141-145 (1988)

7. Çakallı, H: On statistical convergence in topological groups. Pure Appl. Math. Sci. 43, 27-31 (1996)

8. Karakuş, S: Statistical convergence on probabilistic normed spaces. Math. Commun. 12, 11-23 (2007)

9. Alotaibi, A, Alroqi, A: Statistical convergence in a paranormed space. J. Inequal. Appl. (2012). doi:10.1186/1029-242X-2012-39

10. Freedman, AR, Sember, JJ: Densities and summability. Pac. J. Math. 95, 293-305 (1981)

11. Fridy, JA: On statistical convergence. Analysis 5, 301-313 (1985)

12. Connor, JS: The statistical and strong $p$-Cesaro convergence of sequences. Analysis 8, 47-63 (1988)

13. Kızmaz, H: On certain sequence spaces. Can. Math. Bull. 24(2), 169-176 (1981)

14. Et, M, Çolak, R: On some generalized difference sequence spaces. Soochow J. Math. 21(4), 377-386 (1995)

15. Et, M, Nuray, F: $\Delta^{m}$-statistical convergence. Indian J. Pure Appl. Math. 32(6), 961-969 (2001)

16. Et, M: Spaces of Cesaro difference sequences of order $r$ defined by a modulus function in a locally convex space. Taiwan. J. Math. 10(4), 865-879 (2006)

17. Et, M, Choudhary, B, Tripathy, BC: On some classes of sequences defined by sequences of Orlicz functions. Math. Inequal. Appl. 9(2), 335-342 (2006)

18. Mursaleen, M: Elements of Metric Spaces. Anamaya Publishers, New Delhi (2005)

19. Ruckle, WH: FK spaces in which the sequence of coordinate vectors in bounded. Can. J. Math. 25(5), 973-975 (1973)

20. Fridy, JA, Orhan, C: Lacunary statistical convergence. Pac. J. Math. 160(1), 43-51 (1993)

21. Pehlivan, S, Fisher, B: Some sequence spaces defined by a modulus function. Math. Slovaca 45(3), 275-280 (1995)

22. Tripathy, BC, Et, M: On generalized difference lacunary statistical convergence. Stud. Univ. Babeş-Bolyai, Math. 50(1), $119-130(2005)$

doi:10.1186/1029-242X-2013-256

Cite this article as: Altundağ: On generalized difference lacunary statistical convergence in a paranormed space.

Journal of Inequalities and Applications 2013 2013:256.

\section{Submit your manuscript to a SpringerOpen ${ }^{\circ}$ journal and benefit from:}

- Convenient online submission

Rigorous peer review

- Immediate publication on acceptance

Open access: articles freely available online

- High visibility within the field

- Retaining the copyright to your article 